\title{
Chemical Vapor Deposition of Graphene on Platinum: Growth and Substrate Interaction
}

Jungtae Nam ${ }^{1}$, Dong-Chul Kim ${ }^{2,3,}{ }^{*}$, Hoyeol Yun ${ }^{4}$, Dong Hoon Shin ${ }^{5}$, Seungjin Nam ${ }^{6}$, Won Ki Lee ${ }^{6}$, Jun Yeon Hwang ${ }^{6}$, Sang Wook Lee ${ }^{5}$,Helge Weman ${ }^{2,3}$ and Keun Soo Kim ${ }^{1, *}$

${ }^{1}$ Department of Physics and Graphene Research Institute, Sejong University, Seoul, 05006, Republic of Korea

${ }^{2}$ CrayoNano AS, Otto Nielsens vei 12, NO-7052, Trondheim, Norway

${ }^{3}$ Department of Electronics and Telecommunications, Norwegian University of Science and Technology (NTNU), NO-7491, Trondheim, Norway

${ }^{4}$ School of Physics, Konkuk University, Seoul, 05029, Republic of Korea

${ }^{5}$ Department of Physics, Ewha Womans University, Seoul, 03760, Republic of Korea

${ }^{6}$ Institute of Advanced Composite Materials, Korea Institute of Science and Technology, Joellabuk-do, 55324, Republic of Korea

\footnotetext{
* Corresponding author.

E-mail: kskim2676@sejong.ac.kr (Keun Soo Kim)

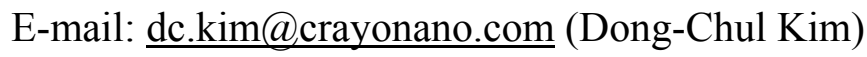




\begin{abstract}
Low-pressure chemical vapor deposition of graphene has been investigated on various Pt substrates such as e-beam deposited films, sputtered films, and polycrystalline foils. High temperature sputtering is found to be crucial in growing single layer graphene on Pt. It gives highly (111)-oriented crystallization with a significant reduction of dewetting in Pt films, in contrast to e-beam deposited Pt films. Graphene grown on high temperature sputtered Pt films is free of micro-sized multilayer graphene islands normally observed in graphene grown on polycrystalline Pt foils. This indicates that using Pt thin films can effectively suppress the multilayer graphene growth by carbon segregations and precipitations from the Pt bulk. Growth of single layer graphene is demonstrated on Pt films with a thickness down to $25 \mathrm{~nm}$. Effects of the Pt substrates on the as-grown graphene have been investigated. An XY plot of the Raman G and 2D bands in graphene shows a correlation with the surface facet orientations of the Pt substrates measured by electron backscatter diffraction. With a general red shift of the $\mathrm{G}$ band distributions, a blue shift of the 2D band distributions is observed, which goes as high as $\sim 2750 \mathrm{~cm}^{-1}$ in graphene grown on $\mathrm{Pt}(111)$ films.
\end{abstract}

\title{
1. INTRODUCTION
}

Chemical vapor deposition (CVD) is the most common method to grow graphene. For CVD growth of graphene metal catalysts such as $\mathrm{Cu}$ and $\mathrm{Ni}$ are generally used, with $\mathrm{Cu}$ as the most popular metal to produce homogeneous single layer graphene in large area. ${ }^{1-3}$ However $\mathrm{Cu}$ contamination on graphene is a fundamental issue which blocks the further usage of graphene for many applications. The $\mathrm{Cu}$ etching and graphene transfer process always leave $\mathrm{Cu}$ remnants on the bottom side of graphene previously attached to $\mathrm{Cu}$ foils. In addition at a typical graphene growth temperature of $900 \sim 1000{ }^{\circ} \mathrm{C}, \mathrm{Cu}$ gets partially melted and sublimated. The generated $\mathrm{Cu}$ vapor easily contaminates the CVD chamber as well as the grown graphene from the top. A recent detailed study on the residual $\mathrm{Cu}$ contamination on asgrown graphene showed its amount could exceed $\sim 10^{13}-10^{14}$ atoms $/ \mathrm{cm}^{2}{ }^{4}$ These residual $\mathrm{Cu}$ impurities are hardly reducible by improving the graphene transfer and cleaning techniques. This $\mathrm{Cu}$ contamination issue poses a big challenge in the integration of CVD grown graphene 
with the front-end Si device manufacturing platforms, which require strict control on the purity of materials used in process. It is also very critical in the use of graphene as an epitaxial substrate for III-V semiconductor growth by metal-organic vapor phase epitaxy (MOVPE) and molecular beam epitaxy (MBE). Especially in in ultra-high vacuum MBE, where ultra-pure sources and substrates with at least $5 \mathrm{~N}$ purity are used for the growth. ${ }^{5} \mathrm{Cu}$ residues in graphene could easily diffuse out at high temperature, and contaminate the grown semiconductor materials, as well as the MBE system itself.

There have been some efforts to grow single layer graphene using metal catalysts other than $\mathrm{Cu}$, which could fundamentally eliminate the contamination issue. Recently several papers on the CVD growth of graphene using Pt as a catalyst have been reported. ${ }^{6-8}$ E.g. millimeter-sized single-crystal graphene has been grown on $\mathrm{Pt}$ foil using ambient pressure CVD. ${ }^{7}$ Low-pressure CVD (LP-CVD) has also been used to grow high-quality graphene on 400 nm-thick Pt films with a pre-annealing at $1000{ }^{\circ} \mathrm{C}$ before the graphene growth. ${ }^{8}$ Compared with $\mathrm{Cu}$, Pt has a much higher melting temperature $\left(\mathrm{T}_{\text {melt }}=1768{ }^{\circ} \mathrm{C}\right.$ for Pt vs. $\mathrm{T}_{\text {melt }}$ $=1084{ }^{\circ} \mathrm{C}$ for $\left.\mathrm{Cu}\right)$ and an almost zero vapor pressure $\left(1.3 \times 10^{-14} \mathrm{mmHg}\right.$ for Pt vs. $6.0 \times 10^{-5}$ $\mathrm{mmHg}$ for $\mathrm{Cu}$ ) at $\mathrm{T}=1000{ }^{\circ} \mathrm{C}$. This makes the contamination from any sublimated Pt during graphene growth negligible in the as-grown graphene. The graphene grown on Pt can be transferred from the Pt surface by electrochemical delamination (ED). ${ }^{7}$ Since the chemically inert Pt does not involve any chemical reactions during the ED process, the transferred graphene will be free of any Pt-related residues. It should be noted that in the ED process with graphene grown on $\mathrm{Cu}$, a chemical etching of $\mathrm{Cu}$ by hydroxides generated at cathode always occurs. The etched $\mathrm{Cu}$ compounds will contaminate the electrolytes and unavoidably also the transferred graphene. ${ }^{4}$

At $\mathrm{T}=1000{ }^{\circ} \mathrm{C}$ the carbon solubility of $\mathrm{Pt}$ is larger $(\sim 0.071 \mathrm{wt} \%)$ than that of $\mathrm{Cu}(<0.002$ wt $\%)$, but smaller than $\mathrm{Ni}(\sim 0.3 \mathrm{wt} \%) .{ }^{9-11}$ This implies that the graphene growth on Pt would be something between a catalytic carbon adsorption process (self-limiting monolayer graphene growth on $\mathrm{Cu}$ ) and a carbon segregation \& precipitation process (multi-layer graphene growth on $\mathrm{Ni}$ ), which would require an optimization of the growth parameters for homogeneous single layer graphene growth on $\mathrm{Pt}^{12}$ Although $\mathrm{Pt}$ is much more expensive than $\mathrm{Cu}$ it can be readily be reused. ${ }^{7,8}$ With a thin film form of $\mathrm{Pt}$ on a $\mathrm{SiO}_{2} / \mathrm{Si}$ support 
substrate, which is more robust than foils which are vulnerable to get wrinkled, the graphene growth on Pt can be easily scaled up to wafer size.

In this paper, we report a thorough study on LP-CVD growth of graphene on Pt. Among various $\mathrm{Pt}$ substrates such as sputter-deposited films, e-beam deposited films and polycrystalline Pt foil, thin Pt films deposited by high temperature sputtering were found to be the best Pt substrate to grow homogeneous, single layer graphene. Successful growth of graphene on Pt films with a thickness down to $25 \mathrm{~nm}$ may be attributed to the highly crystallized Pt (111) structure and its improved adhesion to the bottom $\mathrm{SiO}_{2}$ surface by the high temperature sputtering. In addition, interactions between as-grown graphene and the surface facet orientation of Pt were investigated using electron backscatter diffractions (EBSD) and confocal-Raman mapping. The results are analyzed by plotting the frequencies of the Raman $\mathrm{G}$ and 2D bands in graphene on different facets.

\section{EXPERIMENTAL SECTION}

\subsection{Preparation of Pt substrates \& CVD growth of graphene}

Pt films were prepared on $\mathrm{SiO}_{2}(300 \mathrm{~nm}) / \mathrm{Si}$ substrates either by e-beam evaporation or radio-frequency (RF) magnetron sputtering of a Pt target (99.995\%) at different temperatures (room temperature (RT), $300{ }^{\circ} \mathrm{C}$, and $600{ }^{\circ} \mathrm{C}$ ). Sputtering was done with a RF power of 100 $\mathrm{W}$, a chamber pressure of 5 mTorr with Argon, and a deposition rate of $2 \AA / \mathrm{s}$. E-beam evaporation of $\mathrm{Pt}(99.995 \%)$ was done with a deposition rate of $5 \AA / \mathrm{s}$ in high vacuum $\left(<10^{-4}\right.$ mTorr). Polycrystalline Pt foils (99.99\%, $25 \mu \mathrm{m}$ in thickness, Sigma-Aldrich) were also used.

A LP-CVD system with a movable furnace (Graphene Square Co.) is used to grow graphene on the Pt substrates. For the graphene growth the furnace was first pre-heated to the process temperature of $\sim 1000{ }^{\circ} \mathrm{C}$ with a flow of $\mathrm{H}_{2}$ gas $(50 \mathrm{sccm})$ with the substrate in a position outside the furnace and then moved to the sample position slowly. The sample temperature increased and usually stabilized at $1000{ }^{\circ} \mathrm{C}$ within 7 minutes. After stabilizing the temperature with the substrate in position inside the furnace, the graphene growth started by flowing a $\mathrm{CH}_{4} / \mathrm{H}_{2}$ gas mixture $(10 \mathrm{sccm} / 100 \mathrm{sccm})$. Finally the graphene growth was 
stopped by replacing the gas mixture with Ar gas $(100 \mathrm{sccm})$ and the furnace was moved away immediately from the sample position for fast cooling.

\subsection{Characterization}

The Pt films were characterized by X-ray diffraction (XRD) measurements (SmartLab 9 kW, Rigaku Co.) before and after the graphene growth. Two different Raman setups were used to characterize the graphene. A Renishaw inVia Raman microscope with a $514 \mathrm{~nm}$ laser was used for single spot Raman measurements, after transferring the graphene from the $\mathrm{Pt}$ substrates to $\mathrm{SiO}_{2} / \mathrm{Si}$ substrates by ED. For Raman mapping measurements, an Alpha 300R WiTec confocal-Raman system with a $532 \mathrm{~nm}$ laser was used with a scan step size of 500 $\mathrm{nm}^{7}$ EBSD data of the graphene grown Pt substrates were collected in scanning electron microscopy (Helios Nano-lab 650, FEI Co.) at $15 \mathrm{kV}$ acceleration voltage and $13 \mathrm{nA}$ probe current, and analyzed with EDAX/TSL OIM software. The hexagonal lattice image of graphene was observed in a high resolution transmission electron microscope (HRTEM; TITAN G2 60-300, FEI Co.). To minimize the electron damage during the TEM observation, the acceleration voltage of HRTEM was $80 \mathrm{kV}$.

\section{RESULTS AND DISCUSSION}

\subsection{CVD graphene growth on sputtered Pt film}

In order to grow graphene homogeneously on metal catalyst films such as Pt films, it is absolutely necessary that metal catalyst films should retain its continuous film form during graphene growth at high temperature. Also it should only act as a catalyst for graphene growth without any chemical reaction with underlying substrates at high temperature. These requirements make the sputter deposition of metal catalyst films to be one good choice. Metals with a face-centered-cubic structure such as Pt have the highest sputter yield with a (111) crystal direction, and therefore get deposited with a preferred (111) crystal orientation normal to the substrates in order to minimize the surface energy. ${ }^{13-15}$ High voltage biasing of the target materials for sputtering gives high kinetic energy to the deposited atoms ( 1 to 10 $\mathrm{eV})$ compared to when deposited by evaporation $(\sim 0.1 \mathrm{eV}){ }^{16}$ This results in a better 
adhesion of the deposited metals on the substrate, which is essential in the deposition of a Pt film directly on a $\mathrm{SiO}_{2}$ surface which is chemically inert to $\mathrm{Pt}$ at high temperature for graphene growth. We found that the sputter deposition of $\mathrm{Pt}$ on $\mathrm{SiO}_{2} / \mathrm{Si}$ substrates at elevated temperature gives further improvement in crystallinity of Pt films and adhesion as discussed in the following.

Figure 1 (a) shows XRD data of Pt films (film thickness $(t)=100 \mathrm{~nm}$ ) sputter-deposited at different temperatures, RT, $300{ }^{\circ} \mathrm{C}$, and $600{ }^{\circ} \mathrm{C}$. The (111) crystallization of Pt can be clearly seen in all samples. The heating of the substrates during the deposition gives a large enhancement in the Pt crystallization. The intensity of the $\mathrm{Pt}(111)$ peak of the $300{ }^{\circ} \mathrm{C}$ deposited film increased by more than four times compared to at RT, with a decrease of its FWHM (see Table 1). Further increase in the deposition temperature to $600{ }^{\circ} \mathrm{C}$ showed only a small enhancement in the (111) crystallization of Pt. However after the LP-CVD graphene growth at $\mathrm{T}=1000{ }^{\circ} \mathrm{C}$, with a typical growth time of $10 \mathrm{~min}$, the XRD data changes. In all samples the Pt (111) peak became sharper with an increase of the Pt (111) peak intensity and a decrease of its FWHM value (see Table 1). In addition a Pt (200) peak at $46.5^{\circ}$ appeared. It is clearly noticeable in RT deposited film, while it is almost negligible in the high temperature deposited films. This indicates that the RT deposited Pt films are rather unstable at the high temperature during the graphene growth. The amorphous Pt phase in the RTdeposited films, which is expected to be much larger in portion compared to the high-T deposited films, undergoes crystallization in a way that results in an increase of the Pt (111) peak and an appearance of the Pt (200) phase (which has the second lowest surface energy). ${ }^{14}$ The high temperature deposited Pt films are already well-crystallized in the Pt (111) phase from the sputtering deposition itself. Apart from a moderate enhancement of the Pt (111) peak intensity, it shows little change after the graphene growth. 

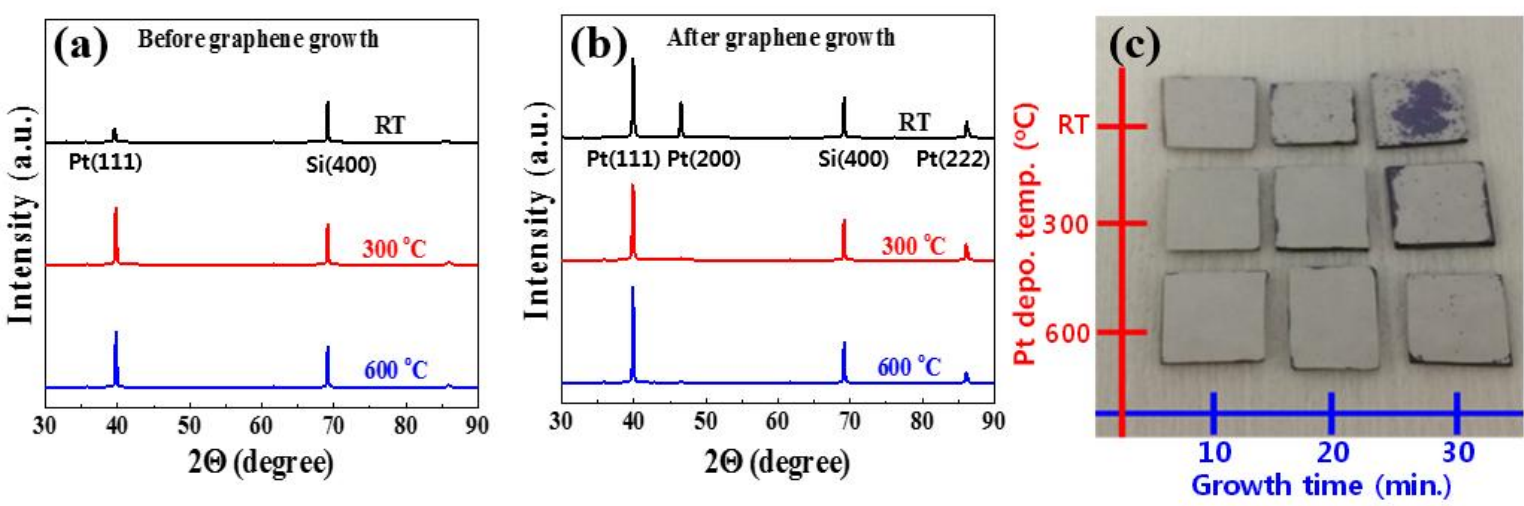

Figure 1. XRD $\Theta-2 \Theta$ scans of Pt films sputter-deposited at different temperatures (RT, 300 ${ }^{\circ} \mathrm{C}$, and $600{ }^{\circ} \mathrm{C}$ ) (a) before and (b) after graphene growth. The XRD data in (a) were measured directly after sputtering. Pt films annealed at high temperature without graphene growth show identical XRD data shown in (b) (see Fig. S2, Supplementary Material). XRD intensity is normalized to have the same intensity of the Si (400) substrate peak in the different scans. (c) Photograph of Pt films after the graphene growth. The $\mathrm{x}-$ and $\mathrm{y}$-axis indicate the graphene growth time and the deposition temperature of the $\mathrm{Pt}$ films, respectively. The thickness of the Pt film is $100 \mathrm{~nm}$ with a typical sample size of $\sim 1 \mathrm{x} 1 \mathrm{~cm}^{2}$.

\begin{tabular}{c|cc|ccc}
\hline \hline & \multicolumn{2}{|c|}{ Before graphene growth } & \multicolumn{3}{|c}{ After graphene growth } \\
\hline $\begin{array}{c}\mathrm{Pt} \\
\text { deposition } \\
\text { temperature } \\
\left({ }^{\circ} \mathrm{C}\right)\end{array}$ & I of Pt (111) & $\begin{array}{c}\text { FWHM of Pt } \\
(111)\end{array}$ & $\begin{array}{c}\text { I of Pt } \\
(111)\end{array}$ & $\begin{array}{c}\text { FWHM of Pt } \\
(111)\end{array}$ & $\begin{array}{c}\text { I of Pt } \\
(200)\end{array}$ \\
\hline RT & 0.377 & 0.280 & 2.302 & 0.108 & 1.028 \\
300 & 1.539 & 0.214 & 2.118 & 0.099 & 0.056 \\
600 & 1.569 & 0.198 & 2.839 & 0.109 & 0.038 \\
\hline
\end{tabular}

Table 1. Intensity (I) and full width at half maximum (FWHM) of the Pt XRD peaks as shown in Figure 1. The intensity of the Pt peaks was normalized to the reference intensity of the $\mathrm{Si}(400)$ peak. 
The difference in the XRD data before and after graphene growth can be related to a dewetting phenomenon of the Pt films observed after the graphene growth. It was found that the RT deposited Pt films were more vulnerable to such dewetting (Fig. 1 (c)). With a growth time of 20 minutes, an exposure of the bottom $\mathrm{SiO}_{2}$ surfaces could be detected due to the dewetting of the Pt at several local spots. After 30 minutes growth time the dewetting prevailed on the whole surface. In the high temperature deposited Pt films some dewetting at the edges in addition to some local spots were observed in the $300{ }^{\circ} \mathrm{C}$ deposited $\mathrm{Pt}$ film after 30 minutes graphene growth time. The $600{ }^{\circ} \mathrm{C}$ deposited $\mathrm{Pt}$ films were found to be more robust without any significant dewetting even after 30 minutes graphene growth. This reveals that a high temperature sputtering is crucial to improve the adhesion of thin Pt films to the $\mathrm{SiO}_{2}$ surface and therefore reduce the dewetting after graphene growth. The observed dewetting in RT deposited Pt films, although its level of crystallization after 10minutes at $1000{ }^{\circ} \mathrm{C}$ is comparable to the high temperature deposited films (from the almost same strong Pt (111) peak in XRD), suggests that the Pt crystal phases formed during the graphene growth do not have a strong adhesion to the $\mathrm{SiO}_{2}$ surface. Such crystal formation involves diffusion of the Pt atoms and this might not happen so easily if the Pt atoms get adhered well to the surface with a high crystalline structure. The adhesion seems to be strong only when the film is sputter-deposited in a highly crystalline (111) Pt phase at high temperature. This gives an important implication on the reusability of the $\mathrm{Pt}$ films. If one wants to use $\mathrm{Pt}$ films repeatedly for graphene growth, it is highly preferable to use sputter-deposited Pt films at high temperature.

The optimization of the CVD process for single layer graphene growth was carried out using $100 \mathrm{~nm}$ thick Pt films sputter-deposited at $\mathrm{T}=600{ }^{\circ} \mathrm{C}$. High quality graphene was obtained with $\mathrm{CH}_{4} / \mathrm{H}_{2}=10 \mathrm{sccm} / 100 \mathrm{sccm}$ at $1000{ }^{\circ} \mathrm{C}$ and a growth time of 10 minutes. Under this growth condition, Pt films deposited at lower temperatures ( $300{ }^{\circ} \mathrm{C}$ and $\left.\mathrm{RT}\right)$ also work well for graphene growth (Fig.2 (a)), implying that the crystallinity of Pt and its slight modification during graphene growth does not affect the graphene growth much. However, it should be noted that single layer graphene can be grown only when the Pt catalyst maintains its crystalline, thin film form without too much dewetting during the growth. If the Pt films get dewetted and discontinuous, the graphene quality degrades with the appearances of a large D peak and a broad 2D peak in the Raman spectra due to a defective multilayer 
graphene formation. This is similar to those observed using e-beam evaporated Pt films, which will be discussed in section 3.3.

Using the same graphene growth parameters, graphene growth on Pt films with different thickness was done. The Pt films were all deposited at $600{ }^{\circ} \mathrm{C}$ in order to have a continuous thin film form without any dewetting. As shown in Fig. 2 (b), single layer graphene can be successfully grown on Pt films with a thickness down to $25 \mathrm{~nm}$ as confirmed by Raman measurements and HRTEM images (Fig. S1, Supplementary Material). Raman spectroscopy is a strong analytical tool for the characterization of carbon-materials. In graphene three prominent Raman peaks - G, 2D, and D peaks - are typically observed. The G peak is located at $\sim 1580 \mathrm{~cm}^{-1}$ and is due to the stretching of the $\mathrm{C}-\mathrm{C}$ bond which is a primary in-plane phonon. The $2 \mathrm{D}$ peak at $\sim 2700 \mathrm{~cm}^{-1}$ is a second-order two-phonon process typical of all kinds of $\mathrm{sp} 2$ carbon systems. The D-peak appears at around $1350 \mathrm{~cm}^{-1}$. It comes from many kinds of defects such as grain boundary, vacancy, edge and so on. The intensity of D peak represents the quality of graphene since it is proportional to the amount of defects in graphene. ${ }^{17-19}$ In addition, one can estimate the number of layers in graphene from the ratio of the $2 \mathrm{D}$ peak intensity to the $\mathrm{G}$ peak intensity. Generally it means a single layer graphene if this value is higher than 2. If it is about 1 (less than 1), it means a bi-layer (few layer) graphene. ${ }^{19}$

Continuous, large area graphene grown on $25 \mathrm{~nm}$ thick Pt films can be delaminated and transferred onto $\mathrm{SiO}_{2} / \mathrm{Si}$ substrates (Fig. 2 (c)). Raman mapping shows decent D/G and 2D/G peak intensity ratios expected from single layer graphene, comparable to those from graphene grown on $200 \mathrm{~nm}$ thick Pt films (Fig.2 (d)). It is observed that the quality of graphene does not show any significant difference depending on the thickness of Pt films in the range of 25 $\mathrm{nm}$ to $200 \mathrm{~nm}$ (see also Table S1, Supplementary material). $25 \mathrm{~nm}$ Pt films seem to be the minimum thickness of high temperature $\left(600{ }^{\circ} \mathrm{C}\right)$ sputter deposited Pt films for graphene growth. $10 \mathrm{~nm}$ thick Pt films did not show any continuity in the Pt layer after graphene growth but instead fine Pt-grained, island-like structures. Broad D and G peaks were observed in Raman spectra of the as-grown graphene (Fig. S3, Supplementary Material). It is worth to mention here that the optimized graphene growth parameters may need a further tuning depending on the Pt thickness. These parameters can be quite different especially with polycrystalline, thick Pt foils $(\mathrm{t}=25 \mu \mathrm{m})$. 

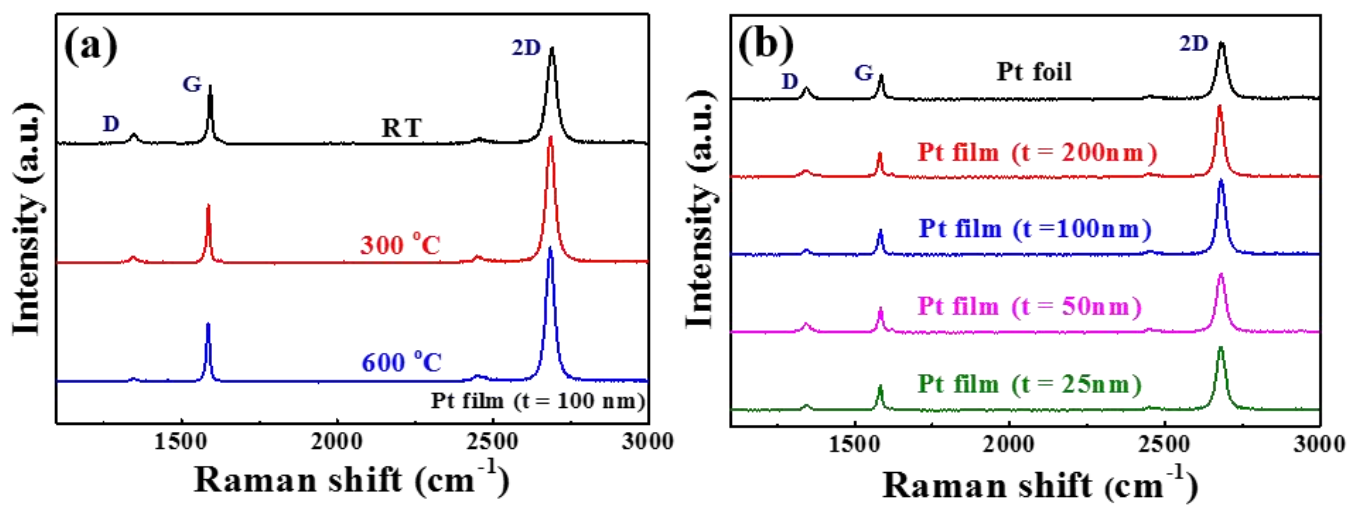

(c) Pt film $(t=25 \mathrm{~nm})$
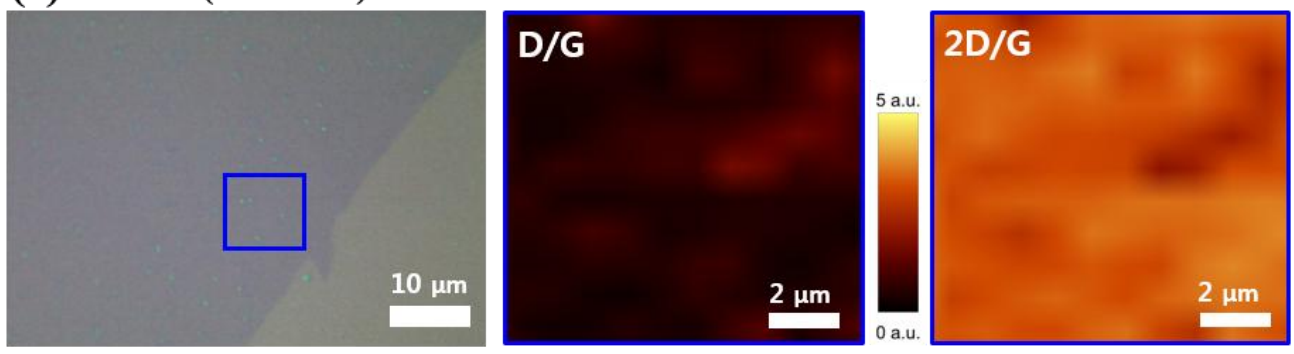

(d) Pt film (t = $200 \mathrm{~nm})$
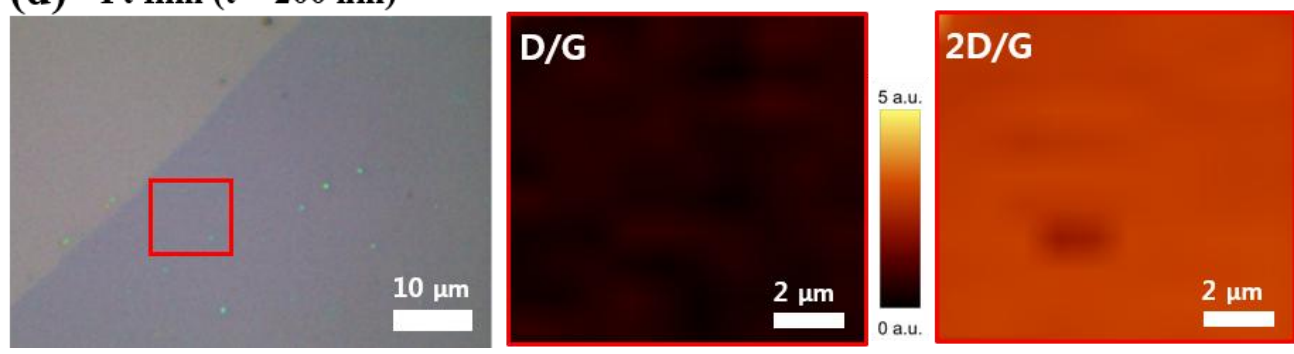

Figure 2. Raman spectra of graphene after transfer onto a $\mathrm{SiO}_{2} / \mathrm{Si}$ substrate. (a) Graphene grown on $100 \mathrm{~nm}$ thick Pt films sputter-deposited at different temperatures (RT, $300{ }^{\circ} \mathrm{C}$ and $600{ }^{\circ} \mathrm{C}$ ). (b) Graphene grown on Pt foil and films with a thickness $\mathrm{t}=25 \mathrm{~nm}, 50 \mathrm{~nm}, 100 \mathrm{~nm}$, and $200 \mathrm{~nm}$, respectively. The same graphene growth parameters $\left(\mathrm{CH}_{4} / \mathrm{H}_{2}=10 / 100\right.$ for 10 min.) were used for all samples. (c) and (d) are the optical microscope images (left) and Raman mapping images (D/G peak intensity ratio (center) and $2 \mathrm{D} / \mathrm{G}$ peak intensity ratio (right)) of graphene grown on Pt films with $\mathrm{t}=25 \mathrm{~nm}$ and $200 \mathrm{~nm}$, respectively. The positions where the Raman mappings were made are denoted as square boxes in the optical microscope images.

\subsection{CVD graphene growth on polycrystalline Pt foil}


Graphene growth on Pt foil was tried with the same growth parameters used in Pt films. Optical microscope image shows dark blue spots scattered randomly on the background of continuous single layer graphene (Fig. 3 (a)). Raman measurements reveal that these are multilayer graphene with $2 \mathrm{D} / \mathrm{G} \leq 1$ and FWHM of $2 \mathrm{D}>40$ (Fig. 3 (b)). Its lateral size is approximately in $\leq 1 \mu \mathrm{m}$ with a number density of $4 \sim 6 \# / 100 \mu \mathrm{m}^{2}$. It is similar to what has been previously observed in graphene growth on $\mathrm{Pt}$ foils. ${ }^{20,21}$ The intermediate carbon solubility of $\mathrm{Pt}$ between $\mathrm{Cu}$ and $\mathrm{Ni}$ makes the absorption of carbon at graphene growth temperature non-negligible. Segregations and precipitations occur and result in a growth of micron-sized multilayer islands or carbon impurities at the Pt surface below earlier formed single-layer graphene. Multilayer graphene islands are hard to be avoided in graphene growth on thick Pt foils.

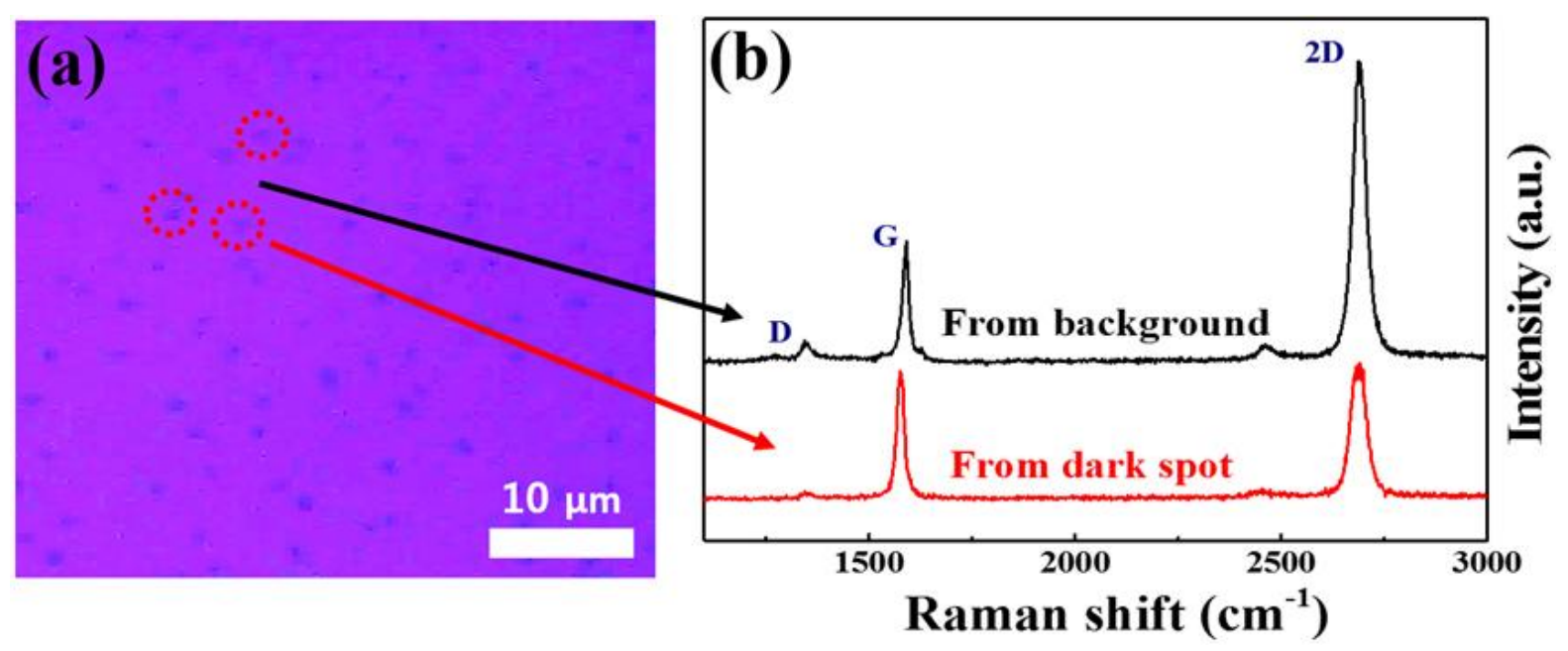

Figure 3. (a) Optical microscope image and (b) Raman spectra of graphene grown on Pt foil after transfer onto a $\mathrm{SiO}_{2} / \mathrm{Si}$ substrate. Raman spectra were taken from background graphene and one of dark spots (red circles) as denoted by the black and red arrow, respectively.

By comparison the growth with Pt films show consistently single layer graphene, even with different $\mathrm{CH}_{4} / \mathrm{H}_{2}$ ratio used (Fig. S4, Supplementary Material). This suggests that the thinness of Pt films would limit the carbon absorption and thus prohibit the multilayer graphene formation from "below" by carbon precipitations and segregations. Similar studies has been done with Ni films where thinner films $(100 \mathrm{~nm})$ with a shorter growth time yield thinner graphene layers compared to thick films $(300 \mathrm{~nm})$ with a longer growth time. ${ }^{2}$ But due to its 
too high carbon solubility suppressing multilayer graphene growth in large area was almost impossible to achieve, which is not the case with Pt films as shown above.

\subsection{CVD graphene growth on e-beam deposited Pt film}

In contrast to sputtered Pt films and Pt foil, the growth of single layer graphene on e-beam deposited Pt films was unsuccessful. Pt is one of the least reactive material and hardly gets oxidized. So the interfacial adhesion of $\mathrm{Pt}$ to $\mathrm{SiO}_{2}$ by forming an oxide layer in-between is very weak. This, together with the absence of a high kinetic energy in sputtering, results in a very poor adhesion of e-beam deposited $\mathrm{Pt}$ films on $\mathrm{SiO}_{2}$. After graphene growth, the Pt films became completely dewetted and coagulated into discontinuous island-like structures, as shown in Fig. 4 (a). The application of a high temperature Pt deposition is also not successful. It was found that even $100 \mathrm{~nm}$ thick Pt films had too weak adhesion and easily peeled off from the $\mathrm{SiO}_{2}$ surface during the preparation of small samples by scribing. The island-like structures are randomly distributed with a height varying from 1 to $10 \mu \mathrm{m}$. Interestingly it is possible to detect graphene-like Raman signals at exposed $\mathrm{SiO}_{2}$ surfaces between the $\mathrm{Pt}$ islands (Fig. 4 (b) and (c)). This means that the graphene structures were formed locally while Pt was still there at the early growth stage. Then later Pt apparently migrated away and left graphene on the $\mathrm{SiO}_{2}$ surface. Although the characteristic $\mathrm{G}$ and 2D peaks from graphene can be observed, the Raman spectra are in general not good and vary significantly from one spot to another with a significant D peak. Dewetted Pt would behave almost like liquid droplets, which do not have stable solid crystal structures. Accordingly, it is expected to have very unstable interactions with carbon at high temperature. Unlike dewetted $\mathrm{Cu}$ films, where single layer graphene can be formed by a self-limiting carbon adsorption process, an unstable carbon absorption followed by segregation and precipitation seem to play a dominant role in dewetted Pt films, producing defective, inhomogeneous graphene. ${ }^{22}$ 

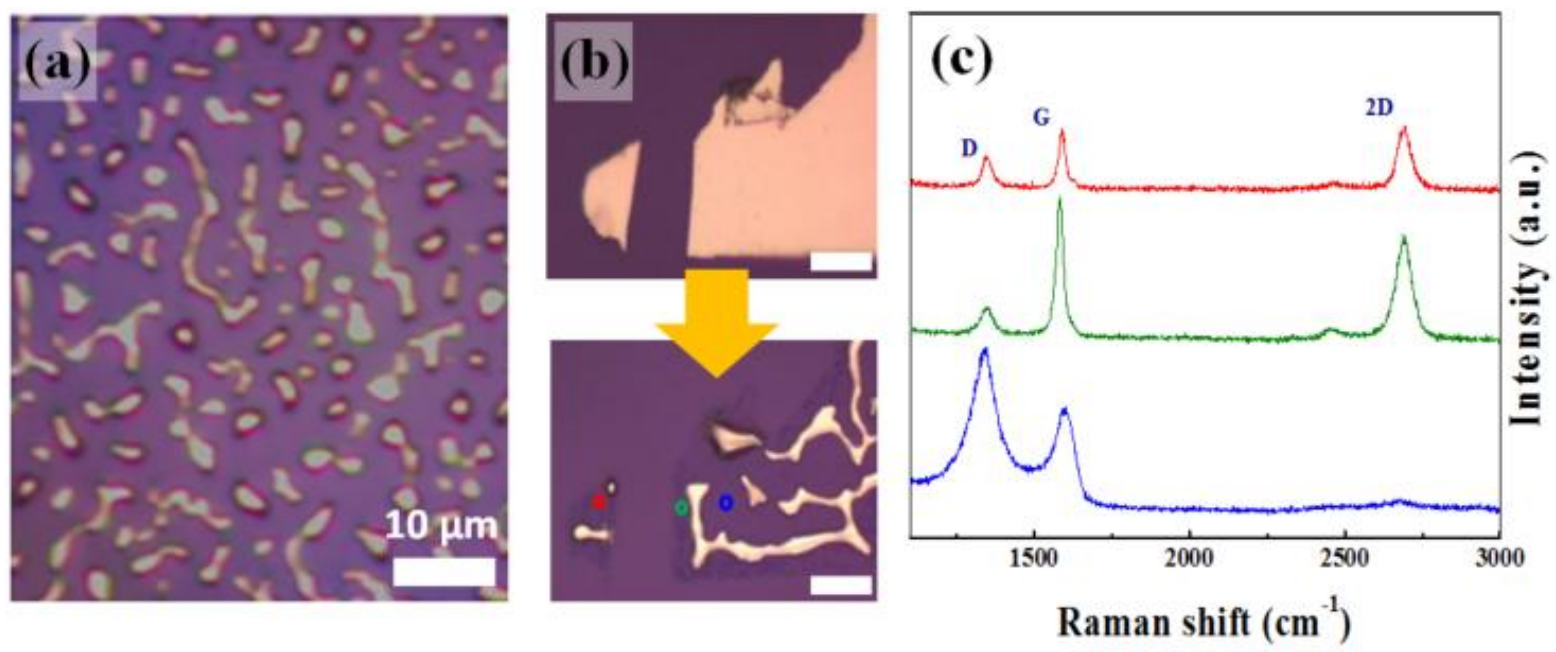

Figure 4. (a) Optical microscope image of an e-beam evaporated Pt film after graphene growth. (b) Change of the optical microscope image of a scratch-patterned e-beam evaporated Pt film before (top) and after graphene growth (bottom). The thickness of the Pt film is $100 \mathrm{~nm}$. (c) Raman spectra from the colored circle positions in the bottom image of (b). The color of the Raman spectra corresponds to that of the circles in (b).

\subsection{Confocal-Raman and EBSD mappings of graphene grown on different crystal} orientations of Pt

Highly crystalline Pt films and Pt foils composed of crystalline domains enable us to investigate the interactions between as-grown graphene and $\mathrm{Pt}$ substrates. The crystal orientation of Pt facets and as-grown graphene on top were checked by EBSD and confocalRaman mapping, respectively. In order to measure the same positions both in EBSD and Raman, marks were made by e-beam lithography (EBL) using PMMA as a negative tone patterns under high dose energy (Fig. S5, Supplementary Material). After EBL and pattern development the samples were rinsed in acetone and isopropanol several times to minimize the resist residues left on the graphene surface. Confocal-Raman mapping was carried out before the EBSD measurements, since the EBSD imaging could change the Raman signals due to hydrocarbon depositions and subtle damages by the e-beam exposure.

Fig. 5 shows confocal-Raman and EBSD mapping results of as-grown graphene on Pt film and Pt foil. EBSD on the Pt film shows a highly textured crystalline structure of Pt (111). 
Each Pt grain is highly (111) crystallized normal to the surface as observed in XRD, but random in in-plane orientation with a grain size of $\leq 1 \mu \mathrm{m}$. The Pt foil is composed of big grains with more than $10 \mu \mathrm{m}$ in size. The grains are almost single crystalline as shown in the inverse pole figure and can be designated with Miller indices. The grain boundaries in the EBSD of Pt foil are clearly observed in Raman mapping data, indicating that the G and 2D band peak frequencies $\left(\omega_{G}\right.$ and $\left.\omega_{2 D}\right)$ of graphene change as the underlying Pt facets change. For better comparisons randomly selected 400 data points from each three facets - (316), (110), and (211) - of the Pt foil are plotted together in addition to those from the Pt (111) film (Fig.5 (c)). The surface energy effect of substrate has been well discussed in graphene grown on copper substrates by Wood et al.. ${ }^{23}$ The (111) plane of Pt substrate showed high quality of graphene in this study, which is well matched with the Wood's observation.

\section{(a) Pt film $(100 \mathrm{~nm})$}
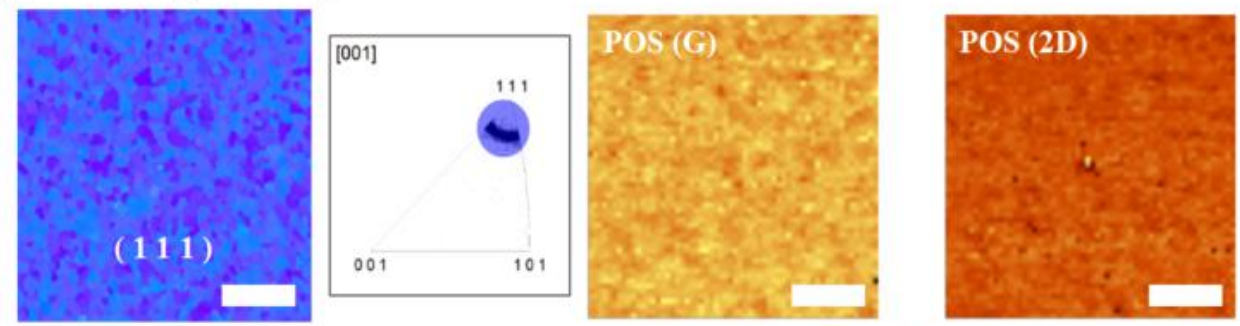

1550

16102640

2780

(b) Pt foil
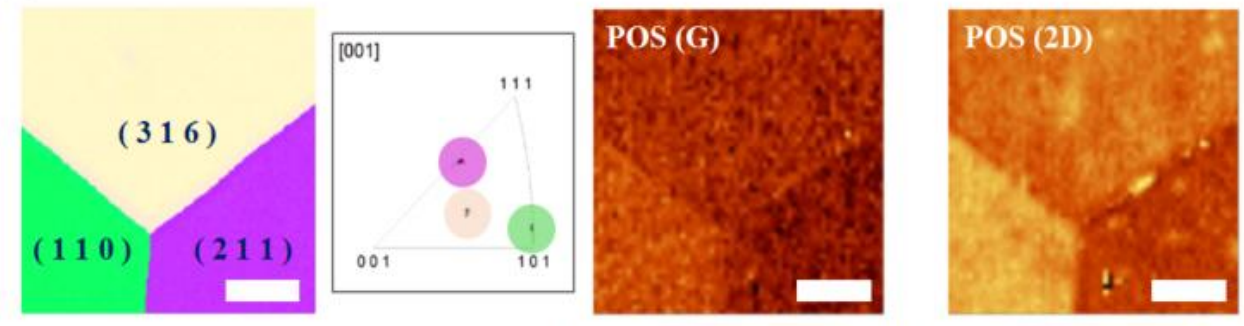

1550

16102640 2700

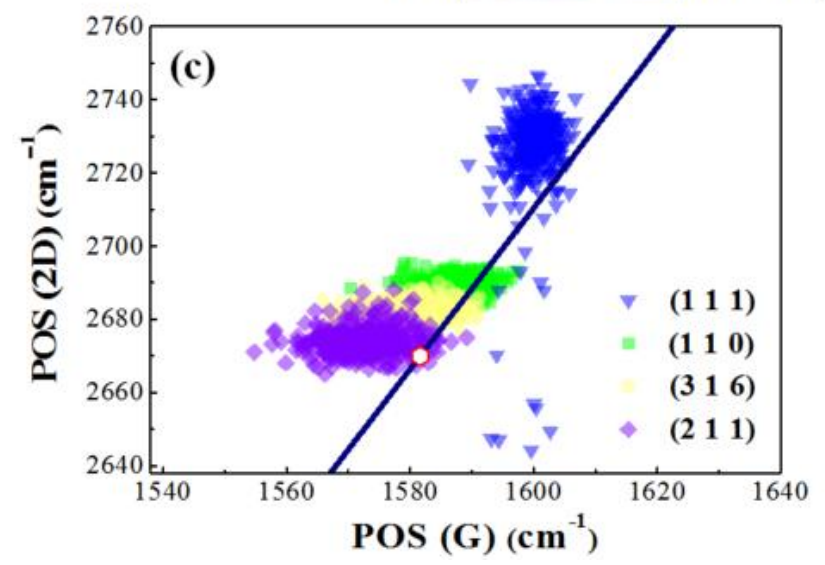


Figure 5. EBSD \& Raman mapping images of as-grown graphene on (a) Pt film and (b) Pt foil. EBSD images and inverse pole figures were taken along the normal direction (ND) to the sample surfaces. Miller indices of the corresponding Pt facets are denoted in the EBSD images. The Raman mapping data show $\omega_{G}$ (POS (G)) and $\omega_{2 D}$ (POS (2D)) of as-grown graphene. All scale bars are $5 \mu \mathrm{m}$. (c) Correlation between POS (G) and POS (2D) of graphene on different Pt facets shown in (a) and (b). The red hexagon on the line is a reference point $\left(\omega_{G}\right.$ and $\omega_{2 D}=1581.6 \mathrm{~cm}^{-1}$ and $\left.2669.9 \mathrm{~cm}^{-1}\right)$ from freestanding, chargeneutral graphene exfoliated from Kish graphite. ${ }^{24}$

Several interesting things can be discussed from Fig. 5 (c). Firstly the G and 2D band peak frequencies of graphene on each facet have a certain distribution which shifts depending on the Pt surface facet orientations. Lee et al. has shown that the effects of strain and charges on graphene can be visualized by correlating the Raman G and 2D bands. ${ }^{24}$ The variation of strain shows a linear behavior with a slope of $\Delta \omega_{2 D} / \Delta \omega_{G}=2.2$ in the POS (2D) vs. POS (G) plot. High-quality graphene samples from Kish graphite deposited on $\mathrm{SiO}_{2}$ were used with a $514.5 \mathrm{~nm}$ laser for this analysis. If this linear correlation is drawn with a shift of $\sim-7 \mathrm{~cm}^{-1}$ in $\omega_{2 D}$ from Lee's paper (the $-7 \mathrm{~cm}^{-1}$ shift with a $532 \mathrm{~nm}$ laser in our case was estimated from the dispersion of $\omega_{2 D}$ with the laser excitation energy, $88 \mathrm{~cm}^{-1} / \mathrm{eV}$ from Mafra et al. ${ }^{25}$ ), one can see that the shift of the distributions from the three facets in the Pt foil looks consistent with the line. It shows a general blue shift with compressive strain, similar to the case for $\mathrm{Cu}^{24-26}$ Secondly, the overall distributions of the $\mathrm{G}$ bands are observed to be somewhat redshifted from the linear line. These red-shifts are mostly located in a region which is "forbidden" in Lee's paper since the doping can only cause blue-shifts of the G bands from the reference point. ${ }^{24,27}$ However, our graphene samples are CVD graphene directly grown on the Pt substrates which is quite different from the case of mechanically exfoliated Kish graphene on $\mathrm{SiO}_{2}$. In fact $\omega_{2 D}$ and $\omega_{G}$ of CVD grown graphene on $\mathrm{Cu}(100)$ and (111) can be also positioned in the forbidden region. ${ }^{28}$ This suggests that the correlation between the $\mathrm{G}$ and 2D bands in CVD graphene on metal substrates can be different from that of exfoliated graphene on $\mathrm{SiO}_{2}$, presumably due to the strong physisorption between graphene and the crystalline metal substrates. It is worth to mention here that the contribution from the 
multilayer spots in graphene grown on the Pt foils to the red-shift of the G band would not be significant. The relative area covered by multilayer spots would be less than $10 \%$ and the expected red-shift from the multilayer graphene would be less than $5 \mathrm{~cm}^{-1}$ with four layers. ${ }^{29}$

Thirdly, data from the Pt (111) film also look consistent with others from the Pt foil as following the linear line. However, the position of their distributions is highly blue-shifted for the $2 \mathrm{D}$ band, which is at $\sim 2730 \mathrm{~cm}^{-1}$ in average. This blue-shift from the reference point $(\sim 60$ $\mathrm{cm}^{-1}$ ) would give a $\sim 0.9 \%$ compressive strain, more than double than that of others. ${ }^{30}$ There have been several reports of Raman measurements on as-grown graphene on Pt (111) crystals and films. ${ }^{6,31,32}$ If the $2 \mathrm{D}$ peak frequencies measured at different laser wavelengths in those papers are converted to the values of our case with $532 \mathrm{~nm}$ laser excitation (under the assumption of a linear shift with the excitation laser wavelength), they would be $\sim 2680$, 2700 and $2713 \mathrm{~cm}^{-1}$, respectively. ${ }^{6,25,31,32}$ All these studies show a blue-shift of the $2 \mathrm{D}$ band from the reference point, although they are not as large as observed in our Pt (111) film, which goes up to $\sim 2750 \mathrm{~cm}^{-1}$. A first-principles study on the graphene-Pt (111) interfaces where a 2 x 2 graphene super cell is adsorbed on Pt (111) surfaces, shows an expansion of the Pt (111) lattice to match a fixed graphene lattice size with a lattice mismatch of $2.5 \%{ }^{33}$ This means that if the Pt (111) lattice is fixed, the graphene lattice would be compressed to match the $\mathrm{Pt}$ (111) lattice. This is consistent to the observed large compressive strain in the graphene on our Pt (111) films.

Finally, we consider any spurious effects from the fabrication process of the mark patterns on the measured Raman data. To estimate this effect Raman mapping was also carried out at a randomly selected spot on as-grown "pristine" graphene on Pt (111) film without such process. This sample shows similar distributions of the $\mathrm{G}$ and $2 \mathrm{D}$ band frequencies to those from the mark-patterned Pt (111) film (see Fig. S6, Supplementary Material). It shows no appreciable blue-shift in the $\mathrm{G}$ band that can be typically caused by p-doping from resist remnants. Therefore it can be concluded that any possible contribution of contaminants from the processing to all the measured Raman mapping data is negligible.

\section{CONCLUSIONS}


In summary, we have investigated the growth of graphene on various types of Pt substrates using low-pressure thermal CVD. It was found that high temperature sputtering is crucial for successful growth of single layer graphene on Pt thin films, which can be attributed to the highly (111)-oriented crystallized structure and high stability of the Pt films without significant dewetting during graphene growth. This enables us to grow single layer graphene on Pt films down to $25 \mathrm{~nm}$ thickness. In contrast to this, e-beam deposited Pt films show large dewetting of the $\mathrm{Pt}$ due to its weak adhesion to the $\mathrm{SiO}_{2} / \mathrm{Si}$ substrates resulting in the growth of defective graphene. Graphene growth on polycrystalline Pt foil results in single layer graphene decorated with micro-sized multilayer graphene islands which were not observed in the graphene grown on high temperature sputtered Pt films. This suggests that $\mathrm{C}$ segregations and precipitations from the Pt bulk can be minimized leading to the growth of homogeneous single layer graphene on the thin Pt films. Raman and EBSD mappings on asgrown graphene show a shift of the Raman $\mathrm{G}$ and 2D bands correlated with the surface facet orientations of the Pt substrates. In graphene grown on the (111)-oriented Pt film a large blueshift of the $2 \mathrm{D}$ band of up to $\sim 2750 \mathrm{~cm}^{-1}$ is observed, indicating a high compressive strain of $\sim 0.9 \%$ in average.

\section{ACKNOWLEDGMENTS}

We acknowledge the financial support from the Research Council of Norway NANO2021 (Grant \#: 228758, Grant \#: 239206) and FORNY2020 (Grant \# 217566, Grant \# 257244) and for the support from the Norwegian Micro- and Nano-Fabrication Facility, NorFab (197411). K. S. Kim acknowledges the Basic Science Research Program through the National Science Foundation of Korea (Grant \#: 2014R1A1A1006414) funded by the Ministry of Science, ICT and Future Planning and Priority Research Centers Program (Grant \#: 20100020207) by the National Research Foundation of Korea (NRF) funded by the Ministry of Education, Science and Technology. This research was also partially supported by Nano. Material Technology Development Program (Grant \#: 2012M3A7B4049888, Grant \#: 2016M3A7B4900135) and by BSR Program (Grant \# 2015R1A2A2A05050829) through the National Research Foundation of Korea (NRF) funded by the Ministry of Science, ICT and Future Planning. 


\section{REFERENCES}

[1] Q. Yu, J. Lian, S. Siriponglert, H. Li, Y. P. Chen, S.-S. Pei, Graphene segregated on Ni surfaces and transferred to insulators, Appl. Phys. Lett. 93 (1) (2008) 113103.

[2] K. S. Kim, Y. Zhao, H. Jang, S. Y. Lee, J. M. Kim, K. S. Kim, et al., Large-scale pattern growth of graphene films for stretchable transparent electrodes, Nature 457 (2009) 706-710.

[3] X. Li, W. Cai, J. An, S. Kim, J. Nah, D. Yang, et al., Large-Area Synthesis of HighQuality and Uniform Graphene Films on Copper Foils, Science 324 (5932) (2009) 13121314.

[4] G. Lupina, J. Kitzmann, I. Costina, M. Lukosius, C. Wenger, A. Wolff, et al., Residual Metallic Contamination of Transferred Chemical Vapor Deposited Graphene, ACS Nano 9 (5) (2015) 4776-4785.

[5] A. M. Munshi, D. L. Dheeraj, V. T. Fauske, D.-C. Kim, A. T. J. Helvoort, B.-O. Fimland, et al., Vertically Aligned GaAs Nanowires on Graphite and Few-Layer Graphene: Generic Model and Epitaxial Growth, Nano Lett. 12 (9) (2012) 4570-4576.

[6] B. J. Kang, J. H. Mun, C. Y. Hwang, B. J. J. Cho, Monolayer graphene growth on sputtered thin film platinum, Applied. Phys. 106 (10) (2009) 104309.

[7] L. Gao, W. Ren, H. Xu, L. Jin, Z. Wang, T. Ma, et al., Repeated growth and bubbling transfer of graphene with millimetre-size single-crystal grains using platinum, Nat. Commun. 3 (2012) 699.

[8] J.-K. Choi, J. Kwak, S.-D. Park, H.-D. Yun, S.-Y. Kim, M. Jung, et al., Growth of Wrinkle-Free Graphene on Texture-Controlled Platinum Films and Thermal-Assisted Transfer of Large-Scale Patterned Graphene, ACS Nano 9 (1) (2015) 679-686.

[9] R. H. Siller, W. A. Oates, R. B. McLellan, The solubility of carbon in palladium and platinum, J. Less-Common Met. 16 (1) (1968) 71-73.

[10] R. B. McLellan, The solubility of carbon in solid gold, copper, and silver, Scripta Metallurgica 3 (6) (1969) 389-391. 
[11] M. Eizenberg, J. M. Blakely, Carbon monolayer phase condensation on Ni(111), Surf. Sci. 82 (1) (1979) 228-236.

[12] X. Li, W. Cai, L. Colombo, R. S. Ruoff, Evolution of Graphene Growth on Ni and Cu by Carbon Isotope Labeling, Nano Lett. 9 (12) (2009) 4268-4272.

[13] G. D. Magnuson, C. E. Carlston, Sputtering Yields of Single Crystals Bombarded by 1to 10-keV Ar ${ }^{+}$Ions, J. Applied. Phys. 34 (11) (1963) 3267.

[14] S. G. Wang, E. K. Tian, C. W. Lung, Surface energy of arbitrary crystal plane of bec and fcc metals, J. Phys. Chem. Solids 61 (8) (2000) 1295-1300.

[15] M. Kawamura, T. Mashima, Y. Abe, K. Sasaki, Formation of ultra-thin continuous Pt and Al films by RF sputtering, Thin Solid Films 377-378 (2000) 537-542.

[16] H. K. Pulker, A. J.Perry, R. Berger, Adhesion, Surf. Tech. 14 (1) (1981) 25-39.

[17] A.C. Ferrari, Raman spcctroscopy of graphene and graphite: Disorder, electron-phonon coupling, doping and nonadiabaitic effects, Solid State Communications, 143 (1-2) (2007) 47-57.

[18] L. G. Cancado, A. Jorio, E. H. Martins Ferreira, F. Stavale, C. A. Achete, R. B. Capaz, et al., Quantifying Defects in Graphene via Raman Spectroscopy at Different Exicatation Energies, Nano Letters, 11 (8) (2011) 3190-3196.

[19] Z. Ni, Y. Wang, T. Yu, Z. Shen, Raman spectroscopy and imaging of graphene, Nano Research, 1 (4) (2008) 273-291.

[20] J. Sun, Y. Nam, N. Lindvall, M. T. Cole, K. B. K. Teo, Y. W. Park, et al., Growth mechanism of graphene on platinum: Surface catalysis and carbon segregation, Appl. Phys. Lett. 104 (15) (2014) 152107.

[21] J. Ping, M. S. Fuhrer, Carbon impurities on graphene synthesized by chemical vapor deposition on platinum, J. Applied. Phys. 116 (4) (2014) 044303. 
[22] A. Ismach, C. Druzgalski, S. Penwell, A. Schwartsberg, M. Zheng, A. Javey, et al., Direct Chemical Vapor Deposition of Graphene on Dielectric Surfaces, Nano Lett. 10 (5) (2010) 1542-1548.

[23] J. D. Wood, S. W. Schmucker, A. S. Lyons, E. Pop, J. W. Lyding, Effects of Polycrystalline $\mathrm{Cu}$ Substrate on Graphene Growth by Chemical Vapor Deposition, Nano Lett. 11 (11) (2011) 4547-4554.

[24] J. E. Lee, G. Ahn, J. Shim, Y. S. Lee, S. Ryu, Optical separation of mechanical strain from charge doping in graphene, Nat. Commun. 3 (2012) 1024.

[25] D. L. Mafra, G. Samsonidze, L. M. Malard, D. C. Elias, J. C. Brant, F. Plentz, et al., Determination of LA and TO phonon dispersion relations of graphene near the Dirac point by double resonance Raman scattering, Phys. Rev. B 76 (2007) 233407.

[26] O. Frank, J. Vejpravova, V. Holy, L. Kavan, M. Kalbac, Interaction between graphene and copper substrate: The role of lattice orientation, Carbon 68 (2014) 440-451.

[27] A. Das, S. Pisana, B. Chakraborty, S. Piscanec, S. K. Saha, U. V. Waghmare, et al., Monitoring dopants by Raman scattering in an electrochemically top-gated graphene transistor, Nat. Nanotech. 3 (2008) 210-215.

[28] R. He, L. Zhao, N. Petrone, K. S. Kim, M. Roth, J. Hone, et al., Large Physisorption Strain in Chemical Vapor Deposition of Graphene on Copper Substrates, Nano Lett. 12 (5) (2012) 2408-2413.

[29] J.-S. Hwang, Y.-H. Lin, J.-Y. Hwang, R. Chang, S. Chattopadhyay, C.-J. Chen, et al., Imaging layer number and stacking order through formulating Raman fingerprints obtained from hexagonal single crystals of few layer graphene, Nanotechnology 24 (1) (2013) 015702. [30] T. M. G. Mohiuddin, A. Lombardo, R. R. Nair, A. Bonetti, G. Savini, R. Jalil, et al., Uniaxial strain in graphene by Raman spectroscopy: G peak splitting, Grüneisen parameters, and sample orientation, Phys. Rev. B 79 (2009) 205433. 
[31] E. Cazzanelli, T. Caruso, M. Castriota, A. R. Marino, A. Politano, G. Chiarello, et al., Spectroscopic characterization of graphene films grown on $\operatorname{Pt}(111)$ surface by chemical vapor deposition of ethylene, J. Raman. Spectrosc. 44 (10) (2013) 1393-1397.

[32] J.-H. Gao, K. Sagisaka, M. Kitahara, M.-S. Xu, S. Miyamoto, D. Fujita, Graphene growth on a Pt (111) substrate by surface segregation and precipitation, Nanotechnology 23 (5) (2012) 055704.

[33] C. Gong, G. Lee, B. Shan, E. Vogel, R. Wallace, K. Cho, First-principles study of metalgraphene interfaces, J. Applied. Phys. 108 (12) (2010) 123711.

\section{LIST OF FIGURES \& TABLES}

Figure 1. XRD $\Theta-2 \Theta$ scans of Pt films sputter-deposited at different temperatures (RT, 300 ${ }^{\circ} \mathrm{C}$, and $600{ }^{\circ} \mathrm{C}$ ) (a) before and (b) after graphene growth. The XRD data in (a) were measured directly after sputtering. The XRD data in (a) were measured directly after sputtering. Pt films annealed at high temperature without graphene growth show identical XRD data shown in (b) (see Fig. S2, Supplementary Material). XRD intensity is normalized to have the same intensity of the Si (400) substrate peak in the different scans. (c) Photograph of Pt films after the graphene growth. The $\mathrm{x}$ - and $\mathrm{y}$-axis indicate the graphene growth time and the deposition temperature of the Pt films, respectively. The thickness of the Pt film is $100 \mathrm{~nm}$ with a typical sample size of $\sim 1 \times 1 \mathrm{~cm}^{2}$.

Figure 2. Raman spectra of graphene after transfer onto a $\mathrm{SiO}_{2} / \mathrm{Si}$ substrate. (a) Graphene grown on $100 \mathrm{~nm}$ thick Pt films sputter-deposited at different temperatures (RT, $300{ }^{\circ} \mathrm{C}$ and $600{ }^{\circ} \mathrm{C}$ ). (b) Graphene grown on Pt foil and films with a thickness t $=25 \mathrm{~nm}, 50 \mathrm{~nm}, 100 \mathrm{~nm}$, and $200 \mathrm{~nm}$, respectively. The same graphene growth parameters $\left(\mathrm{CH}_{4} / \mathrm{H}_{2}=10 / 100\right.$ for 10 min.) were used for all samples. (c) and (d) are the optical microscope images (left) and Raman mapping images (D/G peak intensity ratio (center) and 2D/G peak intensity ratio (right)) of graphene grown on Pt films with $\mathrm{t}=25 \mathrm{~nm}$ and $200 \mathrm{~nm}$, respectively. The positions where the Raman mappings were made are denoted as square boxes in the optical microscope images. 
Figure 3. (a) Optical microscope image and (b) Raman spectra of graphene grown on Pt foil after transfer onto a $\mathrm{SiO}_{2} / \mathrm{Si}$ substrate. Raman spectra were taken from background graphene and one of dark spots (red circles) as denoted by the black and red arrow, respectively.

Figure 4. (a) Optical microscope image of an e-beam evaporated Pt film after graphene growth. (b) Change of the optical microscope image of a scratch-patterned e-beam evaporated Pt film before (top) and after graphene growth (bottom). The thickness of the Pt film is $100 \mathrm{~nm}$. (c) Raman spectra from the colored circle positions in the bottom image of (b). The color of the Raman spectra corresponds to that of the circles in (b).

Figure 5. EBSD \& Raman mapping images of as-grown graphene on (a) Pt film and (b) Pt foil. EBSD images and inverse pole figures were taken along the normal direction (ND) to the sample surfaces. Miller indices of the corresponding Pt facets are denoted in the EBSD images. The Raman mapping data show $\omega_{G}$ (POS (G)) and $\omega_{2 D}$ (POS (2D)) of as-grown graphene. All scale bars are $5 \mu \mathrm{m}$. (c) Correlation between POS (G) and POS (2D) of graphene on different Pt facets shown in (a) and (b). The red hexagon on the line is a reference point $\left(\omega_{G}\right.$ and $\omega_{2 D}=1581.6 \mathrm{~cm}^{-1}$ and $\left.2669.9 \mathrm{~cm}^{-1}\right)$ from freestanding, chargeneutral graphene exfoliated from Kish graphite. ${ }^{24}$

Table 1. Intensity (I) and full width at half maximum (FWHM) of the Pt XRD peaks as shown in Figure 1. The intensity of the Pt peaks was normalized to the reference intensity of the Si (400) peak.

\section{SUPPLEMENTARY MATERIAL}

Seven supporting data are included as below.

Figure S1. (a) HRTEM image of graphene grown on $100 \mathrm{~nm}$ Pt film and fast Fourier transform (FFT) image of (a). The hexagonal honeycomb lattice of carbon atoms and its diffraction pattern of single layer graphene are clearly observed in (a) and (b).

Figure S2. (a) XRD $\Theta-2 \Theta$ scans of "annealed" Pt films sputter-deposited at different temperatures (RT, $300{ }^{\circ} \mathrm{C}$, and $\left.600{ }^{\circ} \mathrm{C}\right)$. “Annealed” means samples have undergone reaching 
and stabilizing at $1000{ }^{\circ} \mathrm{C}$ for 7 minutes with a flow of $\mathrm{H}_{2}$ gas $(50 \mathrm{sccm})$, which is a usual temperature raising and stabilizing process prior to the start of graphene growth in the LPCVD chamber. XRD intensity is normalized to have the same intensity of the Si (400) substrate peak in the different scans. (b) Photograph of Pt films. Reference Pt samples are bare Pt samples directly after sputtering. Annealed Pt samples are samples shown in (a). Graphene grown Pt samples are with graphene grown for 10 minutes after the "annealed" process. Annealed samples as well as graphene grown samples show no difference from reference Pt samples without any dewetting of Pt films.

Figure S3. Optical microscope images of a $10 \mathrm{~nm}$ thick Pt film before (a) and after graphene growth (b). (c) Raman spectra from graphene grown on a $10 \mathrm{~nm}$ thick Pt film. Data were taken from randomly selected different positions in graphene on the Pt film.

Figure S4. Raman spectra of graphene grown with different $\mathrm{CH}_{4} / \mathrm{H}_{2}$ ratios on $100 \mathrm{~nm} \mathrm{Pt}$ films sputter-deposited at $600{ }^{\circ} \mathrm{C}$. Raman spectra were taken after transfer onto a $\mathrm{SiO}_{2} / \mathrm{Si}$ substrate.

Figure S5. Optical microscope images of a (a) graphene/Pt foil and (b) graphene/Pt film after addressing with markers by e-beam lithography (EBL). Crystalline grain domain-boundaries are clearly visible in graphene/Pt foil sample in (b).

Figure S6. Correlation between POS (G) and POS (2D) of graphene on different Pt facets. Data from Raman mapping on "pristine" graphene grown on a Pt (111) film without any process (no resist residues) are added. The blue line is an estimated strain-induced linear variation of $\omega_{G}$ and $\omega_{2 D}$ of graphene from Lee et al. The red hexagon on the line is a reference point $\left(\omega_{G}\right.$ and $\omega_{2 D}=1581.6 \mathrm{~cm}^{-1}$ and $\left.2669.9 \mathrm{~cm}^{-1}\right)$ from freestanding, chargeneutral graphene exfoliated from Kish graphite. ${ }^{24}$ 